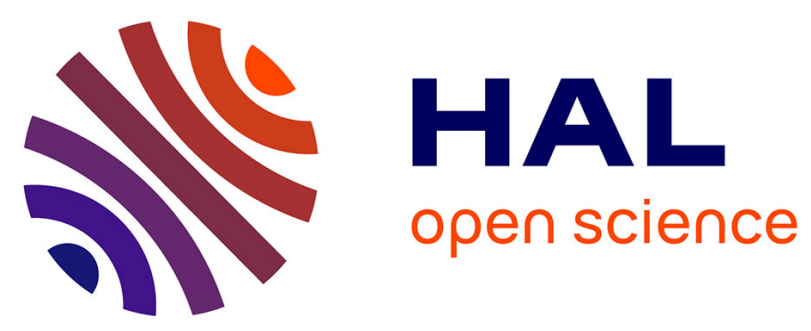

\title{
Fractional Flow Reserve to Guide Treatment of Patients With Multivessel Coronary Artery Disease
}

\author{
Gilles Rioufol, François Dérimay, François Roubille, Thibault Perret, Pascal \\ Motreff, Denis Angoulvant, Yves Cottin, Ludovic Meunier, Laura Cetran, \\ Guillaume Cayla, et al.
}

\section{To cite this version:}

Gilles Rioufol, François Dérimay, François Roubille, Thibault Perret, Pascal Motreff, et al.. Fractional Flow Reserve to Guide Treatment of Patients With Multivessel Coronary Artery Disease. Journal of the American College of Cardiology, 2021, 78 (19), pp.1875-1885. 10.1016/j.jacc.2021.08.061 . hal-03418029

\section{HAL Id: hal-03418029 \\ https://hal.science/hal-03418029}

Submitted on 6 Nov 2021

HAL is a multi-disciplinary open access archive for the deposit and dissemination of scientific research documents, whether they are published or not. The documents may come from teaching and research institutions in France or abroad, or from public or private research centers.
L'archive ouverte pluridisciplinaire HAL, est destinée au dépôt et à la diffusion de documents scientifiques de niveau recherche, publiés ou non, émanant des établissements d'enseignement et de recherche français ou étrangers, des laboratoires publics ou privés. 


\section{Fractional Flow Reserve to Guide Treatment of Patients With Multivessel Coronary Artery Disease}

Gilles Rioufol, MD, PHD, ${ }^{a}$ François Dérimay, MD, PHD, ${ }^{a}$ François Roubille, MD, PHD, ${ }^{b}$ Thibault Perret, MD, ${ }^{c}$ Pascal Motreff, MD, PHD, ${ }^{\mathrm{d}}$ Denis Angoulvant, MD, PHD, ${ }^{\mathrm{e}}$ Yves Cottin, MD, PHD, ${ }^{\mathrm{f}}$ Ludovic Meunier, MD, ${ }^{\mathrm{g}}$ Laura Cetran, MD, ${ }^{\mathrm{h}}$ Guillaume Cayla, MD, PHD, ${ }^{\mathrm{i}}$ Brahim Harbaoui, MD, ${ }^{\mathrm{j}}$ Jean-Yves Wiedemann, MD, ${ }^{\mathrm{k}}$ Éric Van Belle, MD, PHD, ${ }^{1}$ Christophe Pouillot, $\mathrm{MD},{ }^{\mathrm{m}}$ Nathalie Noirclerc, $\mathrm{MD},{ }^{\mathrm{n}}$ Jean-François Morelle, MD, ${ }^{\circ}$ François-Xavier Soto, MD, ${ }^{\mathrm{p}}$ Christophe Caussin, MD, ${ }^{\mathrm{q}}$ Bernard Bertrand, MD, ${ }^{\mathrm{r}}$ Thierry Lefèvre, MD, ${ }^{\mathrm{s}}$ Patrick Dupouy, MD, ${ }^{\mathrm{t}}$ Pierre-François Lesault, MD, PrD, ${ }^{\mathrm{u}}$ Franck Albert, MD, ${ }^{\mathrm{v}}$ Olivier Barthelemy, MD, ${ }^{\mathrm{w}}$ René Koning, MD, ${ }^{\mathrm{x}}$ Laurent Leborgne, MD, ${ }^{\mathrm{y}}$ Pierre Barnay, $\mathrm{MD},{ }^{\mathrm{z}}$ Philippe Chapon, $\mathrm{MD}$, ${ }^{\text {aa }}$ Sébastien Armero, MD, ${ }^{\mathrm{bb}}$ Antoine Lafont, MD, ${ }^{c c}$ Christophe Piot, MD, PHD, ${ }^{\text {dd }}$ Camille Amaz, MSc, ${ }^{\text {ee }}$ Bernadette Vaz, PHARmD, MSc, ${ }^{\text {ee }}$ Lakhdar Benyahya, PнD, ${ }^{\text {ee }}$ Yvonne Varillon, ${ }^{\text {ee }}$ Michel Ovize, MD, PHD, ${ }^{\text {ee }}$ Nathan Mewton, MD, PHD, ${ }^{\text {ee,* }}$ Gérard Finet, MD, PHD, ${ }^{\mathrm{a}, *}$ on behalf of the FUTURE Trial Investigators

\section{ABSTRACT}

BACKGROUND There is limited evidence that fractional flow reserve (FFR) is effective in guiding therapeutic strategy in multivessel coronary artery disease (CAD) beyond prespecified percutaneous coronary intervention or coronary graft surgery candidates.

OBJECTIVES The FUTURE (FUnctional Testing Underlying coronary REvascularization) trial aimed to evaluate whether a treatment strategy based on FFR was superior to a traditional strategy without FFR in the treatment of multivessel CAD.

METHODS The FUTURE trial is a prospective, randomized, open-label superiority trial. Multivessel CAD candidates were randomly assigned (1:1) to treatment strategy based on FFR in all stenotic ( $\geq 50 \%)$ coronary arteries or to a traditional strategy without FFR. In the FFR group, revascularization (percutaneous coronary intervention or surgery) was indicated for FFR $\leq 0.80$ lesions. The primary endpoint was a composite of major adverse cardiac or cerebrovascular events at 1 year.

RESULTS The trial was stopped prematurely by the data safety and monitoring board after a safety analysis and 927 patients were enrolled. At 1-year follow-up, by intention to treat, there were no significant differences in major adverse cardiac or cerebrovascular events rates between groups (14.6\% in the FFR group vs $14.4 \%$ in the control group; hazard ratio: $0.97 ; 95 \%$ confidence interval: $0.69-1.36 ; P=0.85$ ). The difference in all-cause mortality was nonsignificant, $3.7 \%$ in the FFR group versus $1.5 \%$ in the control group (hazard ratio: $2.34 ; 95 \%$ confidence interval: $0.97-5.18 ; P=0.06$ ), and this was confirmed with a 24 months' extended follow-up. FFR significantly reduced the proportion of revascularized patients, with more patients referred to exclusively medical treatment $(P=0.02)$.

From the a Hôpital Cardiologique et Pneumologique Louis Pradel, Hospices Civils de Lyon, Bron, France; 'Hôpital Arnaud de Villeneuve, Montpellier, France; ${ }^{c}$ Centre Hospitalier Saint Joseph-Saint Luc, Lyon, France; ${ }^{\mathrm{d}}$ Hôpital Gabriel Montpied, ClermontFerrand, France; 'EA4245 T2i, Hôpital Trousseau, CHRU de Tours, Université de Tours, Tours, France; ${ }^{\mathrm{f}}$ Hôpital du Bocage Central, Dijon, France; ${ }^{\mathrm{g} C e n t r e ~ H o s p i t a l i e r ~ d e ~ L a ~ R o c h e l l e, ~ L a ~ R o c h e l l e, ~ F r a n c e ; ~ h H o ̂ p i t a l ~ C a r d i o l o g i q u e, ~ C e n t r e ~ H o s p i t a l o-U n i v e r s i t a i r e ~ d e ~}$ Bordeaux, Université de Bordeaux, Bordeaux, France; 'Service de cardiologie, Hôpital Caremeau, Université de Montpellier, Nîmes, France; ${ }^{\mathrm{j}} \mathrm{Hôpital}$ de la Croix-Rousse, Hospices Civils de Lyon, Lyon, France; ${ }^{\mathrm{k}}$ Centre Hospitalier de Mulhouse, Mulhouse, France; ${ }^{1}$ Hôpital Cardiologique Calmette, Lille, France; ${ }^{\mathrm{m}}$ Clinique Sainte Clotilde, La Réunion, France; ${ }^{\mathrm{n}}$ Centre Hospitalier Annecy-Genevois, Annecy, France; ${ }^{\circ} \mathrm{Clinique}$ Saint Martin, Caen, France; ${ }^{\mathrm{P} C e n t r e ~ H o s p i t a l i e r}$ d'Auxerre, Auxerre, France; 


\section{ABBREVIATIONS ANDACRONYMS \\ ACS = acute coronary \\ syndrome \\ CABG = coronary artery bypass grafting \\ $C A D=$ coronary artery disease \\ DSMB = data safety and monitoring board \\ $F F R=$ fractional flow reserve \\ MACCE = major adverse cardiac or cerebrovascular events \\ NSTEMI = non-ST-segment elevation myocardial infarction \\ OMT = optimal medical treatment \\ $\mathrm{PCl}=$ percutaneous coronary intervention}

$\mathrm{R}$ evascularization of the heart of a patient with coronary artery disease (CAD) is indicated when there is a large area of ischemic myocardium, and the clinical benefit increases with the extent of this ischemic area $(1,2)$. On top of medical treatment, the choice of either percutaneous coronary intervention (PCI) or coronary artery bypass grafting (CABG) in multivessel CAD patients also depends on the severity of coronary lesions $(1,3,4)$. Optimal management of these patients hence requires the accurate assessment of the anatomy of the coronary artery lesions and their impact on cardiac perfusion and function.

SEE PAGE 1886

Fractional flow reserve (FFR) has become the gold standard for assessing the functional impact of ischemia-related coronary lesions irrespective of their angiographic aspect (5). Clinical trials have shown that FFR-guided PCI avoids unnecessary treatment of some coronary lesions, reduces the need for urgent revascularization, and improves clinical outcomes in selected groups of singlevessel and multivessel disease patients (6-8). All these studies except one (8) were restricted to patients for whom PCI or CABG was the only therapeutic option for coronary lesions eligible for percutaneous treatment. In this study, FFR-guided management was studied when all treatment options were possible for non-ST-segment elevation myocardial infarction (NSTEMI) patients only (8). Alternatively, it remains unclear whether FFR might help determine the optimal treatment strategy in patients with multivessel CAD at the time of the angiography when all options (PCI, CABG, or medical treatment alone) can be considered.

This trial aimed to assess whether a therapeutic strategy based on FFR is superior to a conventional strategy based on coronary angiography without FFR in reducing the combined rate of death, myocardial infarction, stroke, or unplanned coronary revascularization among patients with multivessel CAD.

\section{METHODS}

STUDY DESIGN. The FUTURE (FUnctional Testing Underlying coronary REvascularization) trial was a prospective randomized, open-label, superiority trial involving all consecutive eligible patients with multivessel CAD at the time of the angiography. It was conducted at 31 centers in France and was approved by an independent Ethics Committee (CPP sud-est IV approval on December 13, 2012) and the French National Agency For Medicines and Health Products Safety (registration on November 2, 2012; number: 2012-A01195-38). The trial was performed following the principles of the Declaration of Helsinki and the European guidelines for Good Clinical Practice.

STUDY POPULATION. CAD patients aged $>18$ years in stable or stabilized condition ST-segment elevation myocardial infarction $>24$ hours after admission, with NSTEMI or unstable angina $>\mathbf{1 2}$ hours and stable angina (maximum Canadian Cardiovascular Society class score I-III), and with atypical chest pain and positive noninvasive stress test and in whom FFR assessment was feasible were eligible for this trial.

Patients had 2- or 3-vessel CAD upon coronary angiography with the presence of a significant lesion (visually assessed diameter of stenosis of $\geq 50 \%$ ) in at least 2 vessels of $\geq 2.5$ - $\mathrm{mm}$ diameter including the left anterior descending coronary artery or 1 stenosis $\geq 50 \%$ on left main coronary artery (2-vessel disease equivalent). Patients with chronic total occlusion in an artery feeding a viable territory were also eligible for the study.

Key exclusion criteria were history of coronary artery surgery, any contraindication to FFR measurement, New York Heart Association functional class IV, and expected survival of $<2$ years. All inclusion and exclusion criteria are detailed

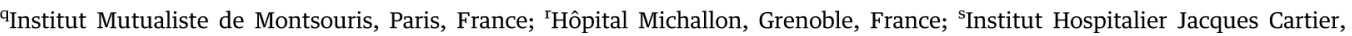
Massy, France; ${ }^{\mathrm{t} C l i n i q u e ~ L e s ~ F o n t a i n e s, ~ M e l u n, ~ F r a n c e ; ~}{ }^{\mathrm{u} H o ̂ p i t a l ~ P r i v e ́ ~ d e ~ l ’ E s t u a i r e, ~ L e ~ H a v r e, ~ F r a n c e ; ~}{ }^{\mathrm{v} C e n t r e ~ H o s p i t a l i e r ~ G e ́ n e ́ r a l, ~}$ Chartres, France; "Hôpital de La Pitié-Salpétrière, Assistance Publique-Hôpitaux de Paris, Paris, France; ${ }^{x}$ Clinique Saint Hilaire, Rouen, France; ${ }^{\mathrm{y}}$ Centre Hospitalier Amiens-Picardie, Hôpital Sud, Amiens, France; ${ }^{\mathrm{z} H o ̂ p i t a l ~ H e n r i ~ D u f f a u t, ~ A v i g n o n, ~ F r a n c e ; ~}$ ${ }^{a a}$ Centre Hospitalier de Valence, Valence, France; ${ }^{b b}$ Hôpital Européen, Marseille, France; ${ }^{c c}$ Hôpital Européen George Pompidou,

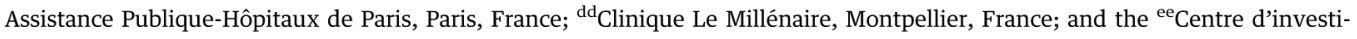
gation clinique de Lyon, INSERM 1407, Hôpital Cardiologique et Pneumologique Louis Pradel, Hospices Civils de Lyon, Bron, France. *Drs Mewton and Finet contributed equally to this work.

The authors attest they are in compliance with human studies committees and animal welfare regulations of the authors' institutions and Food and Drug Administration guidelines, including patient consent where appropriate. For more information, visit the Author Center.
} 
RANDOMIZATION AND MASKING. Patients were randomly assigned (1:1) to 2 different strategy groups: the FFR group or the control group. Randomization was performed via an interactive 24-hour secure voice-response system with a central computerized system. Randomization was stratified according to study site and presence of diabetes. The randomization sequence was concealed. The study was not blinded.

PROCEDURES. In the control group, the decision to treat by PCI, CABG, or medical therapy alone was taken based on the usual angiography estimation of the severity of coronary stenosis, on top of available previous noninvasive tests.

In the FFR group, each coronary lesion with visual estimation of stenosis $\geq 50 \%$ underwent FFR assessment. The study protocol recommended that any coronary stenosis with an FFR $\leq 0.80$ should be treated either by PCI or CABG. In case of chronic total occlusion the FFR value was set at 0.50. Conversely, any coronary stenosis with $\mathrm{FFR}>0.80$ was not treated by PCI or CABG. In both groups, the cardiologist in charge of the patient had the opportunity to propose PCI, CABG, or medical treatment alone. In patients presenting with an acute coronary syndrome (ACS), the culprit coronary artery was revascularized according to guidelines (4). The culprit coronary artery was not included in the multivessel status in STsegment elevation myocardial infarction patients, but it was included in NSTEMI and unstable angina patients

Revascularization procedures by CABG or PCI and modalities of use of pharmacological agents were based on the recent European Society of Cardiology guidelines (4) with the goal of achieving full revascularization. In the control group, patients were treated according to the current best practice. In the FFR group, patients were treated with the intention to revascularize all territories depending on $>2.5 \mathrm{~mm}$ coronary artery and with FFR $\leq \mathbf{0 . 8 0}$. For all procedures, second-generation drug-eluting stents were recommended for PCI while the use of internal mammary arteries was recommended for CABG.

In all cases, it was also recommended to make the final decision after case presentation within a local Heart Team. Best available medical treatment was systematically prescribed, including at least 1 antiplatelet agent, a beta-blocker, an angiotensinconverting-enzyme inhibitor or an angiotensin II receptor blocker, and a statin with a goal to reduce the low-density lipoprotein level to guidelines goals for secondary prevention. Quitting smoking was systematically promoted and diabetes control optimized.
Follow-up on-site visits were scheduled at 1 and 6 months and every year up to 5 years after inclusion. At each visit, the clinical status, electrocardiogram, the presence and severity of angina or dyspnea were recorded, and the quality of life was assessed using the EQ-5D (European Quality of Life 5 Dimensions) questionnaire (9).

Electrocardiographic and coronary angiographic data were stored digitally and sent to the central core laboratory for analysis by blinded experts. Coronary angiography data was scored according to the SYNTAX (SYNergy between PCI with TAXUS and Cardiac Surgery) score algorithm during this central reading (10).

outcomes. The primary endpoint was a composite of death from any cause, nonfatal myocardial infarction, stroke, or unplanned revascularization (ie, revascularization subsequent to the initial strategy that might include a staged procedure) (see Definition Endpoints

within 1 year. Secondary endpoints included individual components of the primary endpoint. Endpoints in this trial were adjudicated by an independent clinical endpoint committee

unaware of the group assignment.

STATISTICAL ANALYSIS. The objective of the study was to investigate the superiority of the FFR-guided strategy over angiography-based therapeutic management. From previous reports $(7,11)$, we estimated that the probability of primary endpoint at 12 months in the control group would be $15.8 \%$. With an anticipated rate of $10 \%$ of patients lost to follow-up, we estimated that 1,728 patients (864 per group) would be required to have $80 \%$ power to detect a $30 \%$ reduction of the risk in the FFR compared with the control group, at a 2-sided type I error rate of 0.05 .

Quantitative variables are described by mean \pm SD or median (interquartile range [IQR]); categorical data are described by absolute and relative frequencies in each category. The comparisons between groups were carried out using the Student's $t$-test or the MannWhitney $U$ test for quantitative characteristics and using the chi-square test or the Fisher exact test for the qualitative characteristics.

All patients were included in the analysis according to the groups to which they were initially assigned (intention-to-treat analysis). A per-protocol analysis of the primary endpoint (assigning patients to the group corresponding to the strategy they received) was also performed. For the primary endpoint, the Kaplan Meier method was used to build the event probability curves and to estimate the event probability at 12 months with its $95 \%$ confidence interval 
TABLE 1 Baseline Demographic, Angiography, and FFR Characteristics in the Intentionto-Treat Population

\begin{tabular}{|c|c|c|c|}
\hline & $\begin{array}{l}\text { Control Group } \\
(\mathrm{n}=\mathbf{4 6 7})\end{array}$ & $\begin{array}{l}\text { FFR Group } \\
(\mathrm{n}=\mathbf{4 6 0})\end{array}$ & $\boldsymbol{P}$ Value $^{\mathrm{a}}$ \\
\hline Age, y & $66 \pm 11$ & $65 \pm 10$ & 0.16 \\
\hline Male & $385(82.0)$ & $393(85.0)$ & 0.22 \\
\hline Body mass index, $\mathrm{kg} / \mathrm{m}^{2}$ & $27 \pm 5$ & $28 \pm 5$ & 0.09 \\
\hline Current smoking & $118 / 460(26.0)$ & $109 / 460(24.0)$ & 0.77 \\
\hline Arterial hypertension & $283(61.0)$ & $265(58.0)$ & 0.33 \\
\hline Dyslipidemia & $286(61.0)$ & $275(60.0)$ & 0.62 \\
\hline Diabetes & $147(32.0)$ & $143(31.0)$ & 0.90 \\
\hline Renal insufficiency ${ }^{b}$ & $180(39.0)$ & $188(41.0)$ & 0.47 \\
\hline Dialysis & $4(1.0)$ & $4(1.0)$ & 0.98 \\
\hline History of myocardial infarction & $100(21.0)$ & $90(20.0)$ & 0.51 \\
\hline History of PCl & $127(27.0)$ & $115(25.0)$ & 0.45 \\
\hline History of stroke & $27(6.0)$ & $13(3.0)$ & 0.03 \\
\hline \multicolumn{4}{|l|}{ Clinical presentation } \\
\hline ACS & $213(46.0)$ & $216(47.0)$ & 0.64 \\
\hline Stable angina ${ }^{c}$ & $102(22.0)$ & $89(19.0)$ & 0.35 \\
\hline Atypical chest pain or silent ischemia & $152(32.0)$ & $155(34.0)$ & 0.71 \\
\hline $\mathrm{CCS} \geq 2$ & $198 / 461(43.0)$ & $175 / 454(39.0)$ & 0.18 \\
\hline Previous noninvasive test & $195 / 465(42.0)$ & $177 / 457(39.0)$ & 0.32 \\
\hline Positive test & 156/195 (80.0) & 142/177 (80.0) & 0.54 \\
\hline LVEF $^{\mathrm{d}}$ & $56 \pm 11$ & $55 \pm 12$ & 0.48 \\
\hline EQ-5D visual analog scale ${ }^{e}$ & $65 \pm 21$ & $66 \pm 19$ & 0.89 \\
\hline \multicolumn{4}{|l|}{ Angiography findings } \\
\hline Radial access & $428(92.0)$ & $412(90.0)$ & 0.28 \\
\hline \multicolumn{4}{|l|}{ Vessels with } \\
\hline 1-vessel disease & $13(3.0)$ & $12(3.0)$ & 0.44 \\
\hline 2-vessel disease & $223(48.0)$ & $201(44.0)$ & \\
\hline 3-vessel disease & $231(50.0)$ & $247(54.0)$ & \\
\hline Left main coronary lesion & $50(11.0)$ & $58(13.0)$ & 0.37 \\
\hline SYNTAX score & $18 \pm 8$ & $19 \pm 8$ & 0.27 \\
\hline \multicolumn{4}{|l|}{ Lesion characteristics } \\
\hline Total number of lesions & 1,634 & 1,632 & \\
\hline $\begin{array}{l}\text { Lesions with stenosis of }>50 \% \text { of } \\
\text { diameter per patient }\end{array}$ & $3(2-4)$ & $3(2-4)$ & 0.50 \\
\hline \multicolumn{4}{|l|}{ FFR findings } \\
\hline Patients with FFR & NA & $450(98.0)$ & \\
\hline FFR failure & NA & $27 / 1,147(2.4)^{f}$ & \\
\hline FFR complication & NA & $9 / 450(2.0)$ & \\
\hline Lesions with FFR (per patient) & NA & $1.38 \pm 1.00$ & \\
\hline Mean FFR & NA & $0.77 \pm 0.13$ & \\
\hline Lesions with FFR $>0.80$ & NA & $470 / 1,090(43.0)$ & \\
\hline Mean FFR in lesions with FFR $\leq 0.80$ & NA & $0.68 \pm 0.11$ & \\
\hline Mean FFR in lesions with FFR $>0.80$ & NA & $0.88 \pm 0.05$ & \\
\hline
\end{tabular}

Values are mean $\pm \mathrm{SD}, \mathrm{n}(\%), \mathrm{n} / \mathrm{N}(\%)$, or median (interquartile range). There were no significant differences between the 2 randomly assigned groups in any of the baseline characteristics, except for history of stroke $(P=0.03)$ and $50 \%$ to $69 \%$ diameter stenosis frequency $(P=0.0007)$. ${ }^{\mathrm{a}}$ The $P$ values are for the FFR group as compared with the control group of patients. ${ }^{b}$ Renal insufficiency was defined as a glomerular filtration rate of $<60 \mathrm{~mL} / \mathrm{min}$. 'Angina was classified according to the CCS functional classification, in which classes range from I to IV, with higher classes indicating greater limitations on physical activity owing to angina. ${ }^{\mathrm{d}} \mathrm{LVEF}$ as defined by standard cardiac ultrasound, or any other noninvasive imaging modality. ${ }^{e}$ EuroQol group score. ${ }^{f} \mathrm{~A}$ total of 1,090 of 1,632 lesions had an FFR measure performed. Culprit lesions for non-ST-segment elevation myocardial infarction and unstable angina and total occluded lesions were not assessed as per protocol. Only 27 lesions were not measured for anatomical or technical reasons.

ACS = acute coronary syndrome; CCS = Canadian Cardiovascular Society; EQ-5D = European Quality of Life 5 Dimensions; FFR = fractional flow reserve; LVEF = left ventricular ejection fraction; NA = not available; $\mathrm{PCI}$ = percutaneous coronary intervention; SYNTAX = SYNergy between PCI with TAXUS and Cardiac Surgery.
(CI) in each group. The log-rank test was used to compare the event probability curves between groups. A Cox regression model stratified on the center and adjusted on the diabetes status was carried out to estimate the hazard ratio (HR) of the FFR compared with the control group with its 95\% CI. The same strategy was carried out for the analysis of each component of the primary endpoint and the analysis of the primary endpoint in different subgroups of patients.

All the reported subgroup analyses were specified before database lock and are listed in the statistical analysis plan. Our institution statistical department performed all analyses with the use of the statistical software SAS, version 9.3 (SAS Institute).

An additional exploratory analysis was performed to assess the predictors of major adverse cardiac or cerebrovascular events (MACCEs) in our study population among the principal baseline clinical and angiography characteristics. An univariate logistic regression followed by a multivariate analysis including all variables that were significantly associated with MACCEs was performed. This trial was registered with ClinicalTrials.gov (NCT01881555).

ROLE OF THE FUNDING SOURCE. G.R., N.M., M.O., and G.F. had full access to all the data in the study and together with the steering committee made the final decision to submit for publication. An independent data safety and monitoring board (DSMB) oversaw the trial.

\section{RESULTS}

SAFETY ANALYSIS. After enrollment of a total of 941 patients, a safety analysis including all patients randomized was performed and showed a significantly higher all-cause mortality rate in the FFR group compared with the control group. Upon the DSMB recommendation , the steering committee decided to stop recruitment and to perform a 1-year follow-up for all patients randomized in the study.

In this safety analysis, the rate of death at 12 months was $20(4.3 \%)$ of 469 patients in the FFR group and $8(1.8 \%)$ of 472 patients in the control group (hazard ratio: 2.39; 95\% confidence interval: 1.05-5.43; $P=0.038)$

There were $10(2.2 \%)$ complications related to the FFR procedure, including 4 FFR guide-induced coronary artery dissections (including 1 case fatality), 3 
guide pressure dysfunction or fractures, and 3 transient atrial arrhythmias or severe chest pain during adenosine administration.

INTENTION-TO-TREAT ANALYSIS. After the premature stop of inclusions following the DSMB recommendation, 927 of the 941 patients were included in the intention-to-treat analysis, 9 patients in the FFR group and 5 in the angiography group having withdrawn consent and 1 patient in the angiography group being lost to follow-up

All

927 patients had a 2- or 3-vessel disease, with 460 patients assigned to the FFR group and 467 patients assigned to the control group. The median duration of follow-up was comparable in both groups with a median follow-up of 24 months (IQR: 12.5-37.1 months) $(P=0.86)$. Only $1(0.1 \%)$ patient was lost to follow-up, and 10 (1.0\%) patients had missing informed consent or withdrawal of consent. At 1 year and also at a mean $24 \pm 12$ months' follow-up, there was no significant difference in mortality between the 2 groups Owing to budgetary restrictions, we were unable to extend followup further.

The 2 groups were well balanced concerning the baseline characteristics (Table 1). The number of patients with a history of a previous stroke was however significantly higher in the control group. More than $30.0 \%$ of the patients had diabetes, and $40.0 \%$ had angina of class II to IV. Approximately $45.0 \%$ of patients were included following an ACS. A total of $19.0 \%$ of ACS patients in the control group and $20.0 \%$ in the FFR group were included after ST-segment elevation myocardial infarction managed by primary angioplasty during the previous days, and were reassessed for multivessel coronary status, over and above the culprit lesion treated in acute phase and not included in the analysis. The coronary angiography characteristics were similar between groups.

There was a significant difference in the therapeutic strategy between the 2 groups. While the proportion of CABG revascularization was similar between groups, more patients were referred to medical treatment alone and less were referred to revascularization by PCI in the FFR group compared with the control group $(P=0.002)$ (Table 2 , Figure 1,

In PCI-treated patients, the revascularization procedure was comparable between the 2 groups. In patients treated by PCI, the mean SYNTAX score was significantly higher in the FFR group compared with the control group $(P=0.007)$. Conversely, in patients treated by CABG, the mean SYNTAX score was higher in the control group compared with the FFR group

\begin{tabular}{|c|c|c|c|}
\hline & $\begin{array}{l}\text { Control Group } \\
(n=467)\end{array}$ & $\begin{array}{l}\text { FFR Group } \\
(n=460)\end{array}$ & P Value $^{a}$ \\
\hline Revascularization strategy & & & 0.002 \\
\hline Optimal medical treatment only & $43(9.0)$ & $78(17.0)$ & \\
\hline CABG & $55(12.0)$ & $54(12.0)$ & \\
\hline $\mathrm{PCl}$ & $369(79.0)$ & $328(71.0)$ & \\
\hline \multicolumn{4}{|l|}{$\mathrm{PCl}$} \\
\hline Lesions with $50 \%-70 \%$ stenosis & $517(42.7)$ & $548(49.5)$ & 0.94 \\
\hline Lesions with CTO & $55(4.5)$ & $63(6.0)$ & 0.96 \\
\hline 3-vessel disease patients & $258(69.9)$ & $247(75.3)$ & 0.13 \\
\hline SYNTAX score & $17 \pm 7$ & $19 \pm 8$ & 0.007 \\
\hline Stents per patient & $2.2 \pm 1.2$ & $2.1 \pm 1.2$ & 0.54 \\
\hline Drug-eluting stents & $745(94.0)$ & $657(95.0)$ & \\
\hline Complete revascularization & $148(57.4)$ & $135(54.7)$ & 0.84 \\
\hline \multicolumn{4}{|l|}{ CABG } \\
\hline Lesions with $50 \%-70 \%$ stenosis & $87(40.3)$ & $90(40.2)$ & 0.96 \\
\hline Lesions with Сто & $21(10.0)$ & $23(10.0)$ & 0.97 \\
\hline 3-vessel disease patients & $48(13.5)$ & $51(15.9)$ & 0.33 \\
\hline SYNTAX score & $26 \pm 9$ & $24 \pm 6$ & 0.034 \\
\hline Mean of total anastomoses & $2.9 \pm 0.9$ & $2.9 \pm 0.9$ & 0.81 \\
\hline Mean of arterial anastomoses & $2.3 \pm 0.9$ & $2.2 \pm 0.9$ & 0.40 \\
\hline Complete revascularization & $30(62.5)$ & $30(58.8)$ & 1 \\
\hline \multicolumn{4}{|l|}{ Optimal medical treatment } \\
\hline Lesions with $50 \%-70 \%$ stenosis & $90(70.0)$ & $164(72.0)$ & 0.15 \\
\hline Lesions with Сто & $10(6.8)$ & $13(5.4)$ & 0.72 \\
\hline SYNTAX score & $17 \pm 9$ & $16 \pm 7$ & 0.56 \\
\hline
\end{tabular}

Values are $\mathrm{n}(\%)$ or mean $\pm S D$. There were significant differences between the 2 randomly assigned groups in the revascularization strategy, with more patients treated by $\mathrm{PCl}$ in the control group compared with the FFR group and fewer patients treated with best medical therapy only in the control group compared with the FFR group $(P=0.002)$. ${ }^{\text {TT }}$ The $P$ values are for the FFR group as compared with the control group of patients.

CABG $=$ coronary artery bypass grafting; $\mathrm{CTO}=$ chronic total occlusion; other abbreviations as in Figure 1.

$(P=0.034)$ (Table 2). Despite finding FFR $>0.80,127$ (11.5\%) lesions were managed by PCI and stenting; likewise, 38 (16.9\%) bypass grafts were performed.

At 1-year follow-up, 134 (14.4\%) patients had at least 1 primary endpoint event. In the intention-totreat analysis, we observed no significant difference for the primary endpoint of MACCE between the FFR group with $14.6 \%$ (67 events) and the control group with $14.4 \%$ (67 events) (HR: 0.97; 95\% CI: 0.69-1.36; $P=0.85$ ) (Table 3, Figure 2). This absence of difference between groups was sustained in the long-term survival analysis with a median follow-up of 24 months (IQR: 12.5-37.1 months) (Figure 2).

In the intention-to-treat analysis, all-cause mortality was $3.7 \%$ in the FFR group versus $1.5 \%$ in the control group (HR: $2.34 ; 95 \% \mathrm{CI}: 0.97-5.18 ; P=0.06$ )

Cardiovascular mortality was $2.6 \%$ in the FFR group versus $1.1 \%$ in the control group (HR: 2.37 ; 95\% CI: $0.83-6.76 ; P=0.11$ ). FFR patients treated by PCI who died within 12 months had more often chronic total occlusion, 3-vessel disease, and a higher SYNTAX score than patients who died in the control group 
FIGURE 1 Revascularization Strategy in the Intention-to-Treat Population

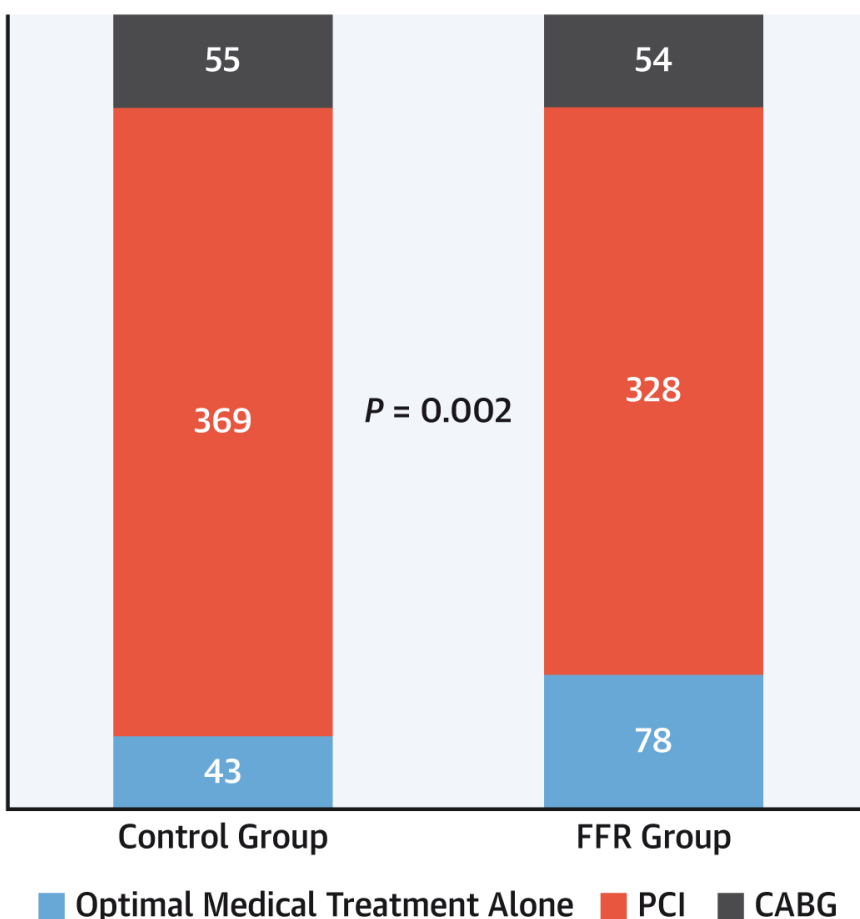

There were 467 patients randomized in the control group and 460 patients randomized in the fractional flow reserve (FFR) strategy group $(n=927)$. The strategy's changes are according to study group. $\mathrm{CABG}=$ coronary artery bypass grafting; $\mathrm{PCl}=$ percutaneous coronary intervention.

At 1-year of follow-up, myocardial infarction was $6.1 \%$ in the FFR group versus $6.0 \%$ in the control group (HR: 1.03; 95\% CI: 0.61-1.74; $P=0.90$ ), stroke was $0.2 \%$ in the FFR group versus $1.5 \%$ in the control group (HR: 0.13; 95\% CI: 0.02-1.07; $P=0.06$ ), and unplanned revascularization was $8.0 \%$ in the FFR group versus $9.9 \%$ in the control group (HR: 0.79 ; 95\% CI: 0.51-1.22; $P=0.28$ ).

\begin{tabular}{|c|c|c|c|c|}
\hline Events & $\begin{array}{l}\text { Control Group } \\
(n=467)\end{array}$ & $\begin{array}{l}\text { FFR Group } \\
(n=460)\end{array}$ & HR $(95 \% \mathrm{Cl})$ & P Value ${ }^{a}$ \\
\hline $\begin{array}{l}\text { Composite of death from any cause, } \\
\text { myocardial infarction, stroke, } \\
\text { and unplanned revascularization }\end{array}$ & $67(14.4)$ & $67(14.6)$ & $0.97(0.69-1.36)$ & 0.85 \\
\hline Death from any cause & $7(1.5)$ & $17(3.7)$ & $2.34(0.97-5.68)$ & 0.06 \\
\hline Cardiovascular death & $5(1.1)$ & $12(2.6)$ & $2.37(0.83-6.76)$ & 0.11 \\
\hline Myocardial infarction & $28(6.0)$ & $28(6.1)$ & $1.03(0.61-1.74)$ & 0.90 \\
\hline Stroke & $7(1.5)$ & $1(0.2)$ & $0.13(0.02-1.07)$ & 0.06 \\
\hline Unplanned revascularization & $46(9.9)$ & $37(8.0)$ & $0.79(0.51-1.22)$ & 0.28 \\
\hline
\end{tabular}

Values are $n(\%)$ unless otherwise indicated. All results were adjusted for center and diabetes status (initial stratification at randomization). The HR that is presented is in consequence adjusted for these 2 variables. ${ }^{\text {a }}$ The $P$ values are for the FFR group as compared with the control group of patients.

$\mathrm{Cl}=$ confidence interval; FFR $=$ fractional flow reserve; $\mathrm{HR}=$ hazard ratio.
In the prespecified subgroup analysis, there was a trend toward higher rates of MACCE in patients with SYNTAX score $>32$ in the FFR group 10 (43.4\%) of 23 events compared with the control group 3 (10.3\%) of 29 events (HR: 3.3; 95\% CI: $0.66-$ $\left.16.8 ; P_{\text {interaction }}=0.15\right)$. HRs and CIs for the primary composite endpoint by prespecified subgroups are reported in Figure 3.

There was no significant difference between the 2 study groups in quality-of-life scores at 1 year (visual scale $=71 \pm 19$ in the FFR group vs $71 \pm 16$ in the control group; $P=0.62$ ).

To further analyze the influence of FFR on clinical outcomes at 1 year, we performed a univariate and a multivariate analysis and examined the association of patients' characteristics with the study primary outcome . We found that a left ventricular ejection fraction $<40 \%$ (HR: 2.33; 95\% CI: 1.07-4.74; $P=0.02$ ) and a SYNTAX score $>32$ (HR: 3.36; 95\% CI: 1.39-7.65; $P<0.001$ ) were independently associated with the combined incidence of MACCE.

\section{DISCUSSION}

The FUTURE trial is one of the few randomized, openlabel prospective trials performed in "all-comer" multivessel CAD patients, assessing all available treatment strategies (optimal medical treatment [OMT], PCI, and CABG) and comparing FFR measurement versus traditional management without FFR. The study was prematurely stopped following a recommendation of the independent DSMB that observed significantly higher all-cause mortality in patients allocated to the FFR group. However, this observation was not confirmed by the intention-totreat analysis at 1-year follow-up. At 1-year followup, there was no significant difference between the FFR-based strategy and a traditional angiographic strategy without FFR for the primary endpoint of MACCE.

The higher all-cause mortality associated with the FFR strategy in our safety analysis was, in our view, due to chance. The absolute number of deaths between groups was in fact very small; the fragility index for this significant difference is low, with a value of 1 (12). A small change in event counts would make the difference nonsignificant. And this is indeed what we observed when shifting from the safety population to the intention-to-treat population at $\mathbf{1}$ year of follow-up, and this was confirmed in the extended follow-up. Even so, the odds ratios were more or less identical on safety analysis and for intention to treat, and it cannot be completely ruled 


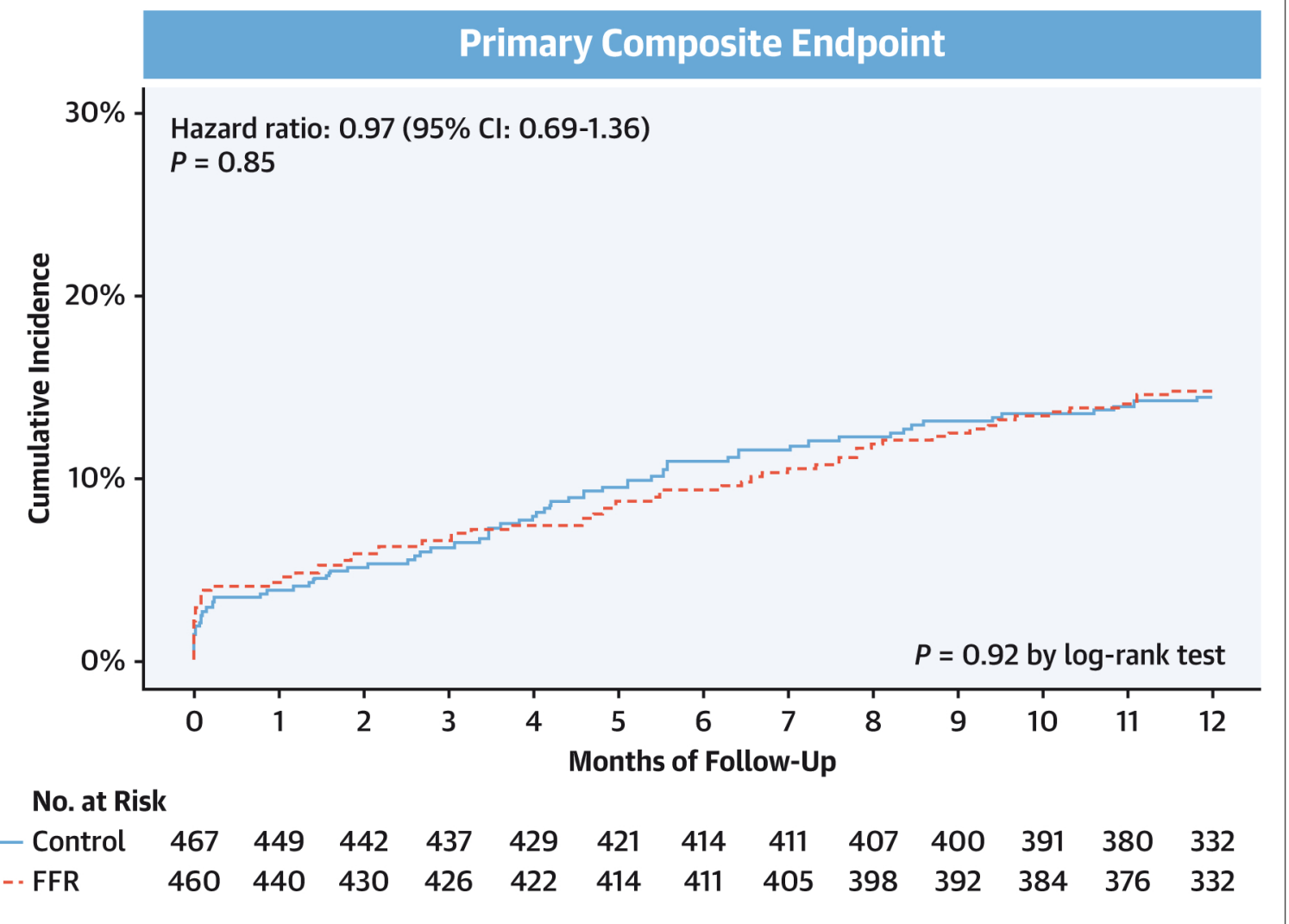

Kaplan-Meier curves are shown for the cumulative incidence of composite primary endpoint of death from any cause, nonfatal myocardial infarction, stroke, or unplanned revascularization in the group that was randomly assigned to a fractional flow reserve (FFR)-based strategy (FFR group) and the group that was randomly assigned to an angiography alone-based strategy (control group). In this intention-to-treat analysis, the rate of primary endpoint at 12 months was 67 (14.6\%) of 460 in the FFR group and 67 (14.4\%) of 467 in the control group. $\mathrm{Cl}=$ confidence interval.

out that there was an effect of FFR-based treatment strategy. The hypothesis that FFR may induce a bias toward immediate intervention (ie, PCI), rather than toward surgical revascularization, cannot, at this point, be excluded.

Even if this difference was not due to chance, there are no data suggesting that it was related to performing FFR measurement except in 1 case in which left main dissection occurred during the procedure

With a $97.7 \%$ success rate of FFR measurement on targeted lesions, our results are in line with those reported in randomized FFR trials or registries $(7,8,13,14)$. The rate of serious complications associated with FFR measurement ( $\mathrm{n}=4$ of 464 , $0.8 \%$ ) was comparable to the $0.2 \%$ and $2 \%$ rates reported in the FAMOUS-NSTEMI (Fractional flow reserve versus angiography in guiding management to optimize outcomes in non-ST-elevation myocardial infarction) and RIPCORD (Does Routine Pressure Wire
Assessment Influence Management Strategy at Coronary Angiography for Diagnosis of Chest Pain) studies, respectively, and lower than the $4 \%$ rate in the ORBITA (Objective Randomised Blinded Investigation with optimal medical Therapy of Angioplasty in stable angina) trial $(8,13,15)$.

In a clinical setting including PCI with secondgeneration drug-eluting stents, predominantly 2-artery grafts for CABG, and high rates of OMT, our study failed to show a significant benefit in terms of MACCE at 1 year with an invasive FFR-based strategy compared with the traditional angiography strategy. There are few randomized controlled trials comparing FFR-based versus traditional angiography revascularization strategies in CAD patients. The FAME (Fractional Flow Reserve versus Angiography for Multivessel Evaluation) study, in stable angina or stabilized ACS, showed significant clinical benefit on MACCE at 1 year in favor of an FFR-based strategy 
FIGURE 3 Subgroup Analyses for the Primary Endpoint at 1 Year

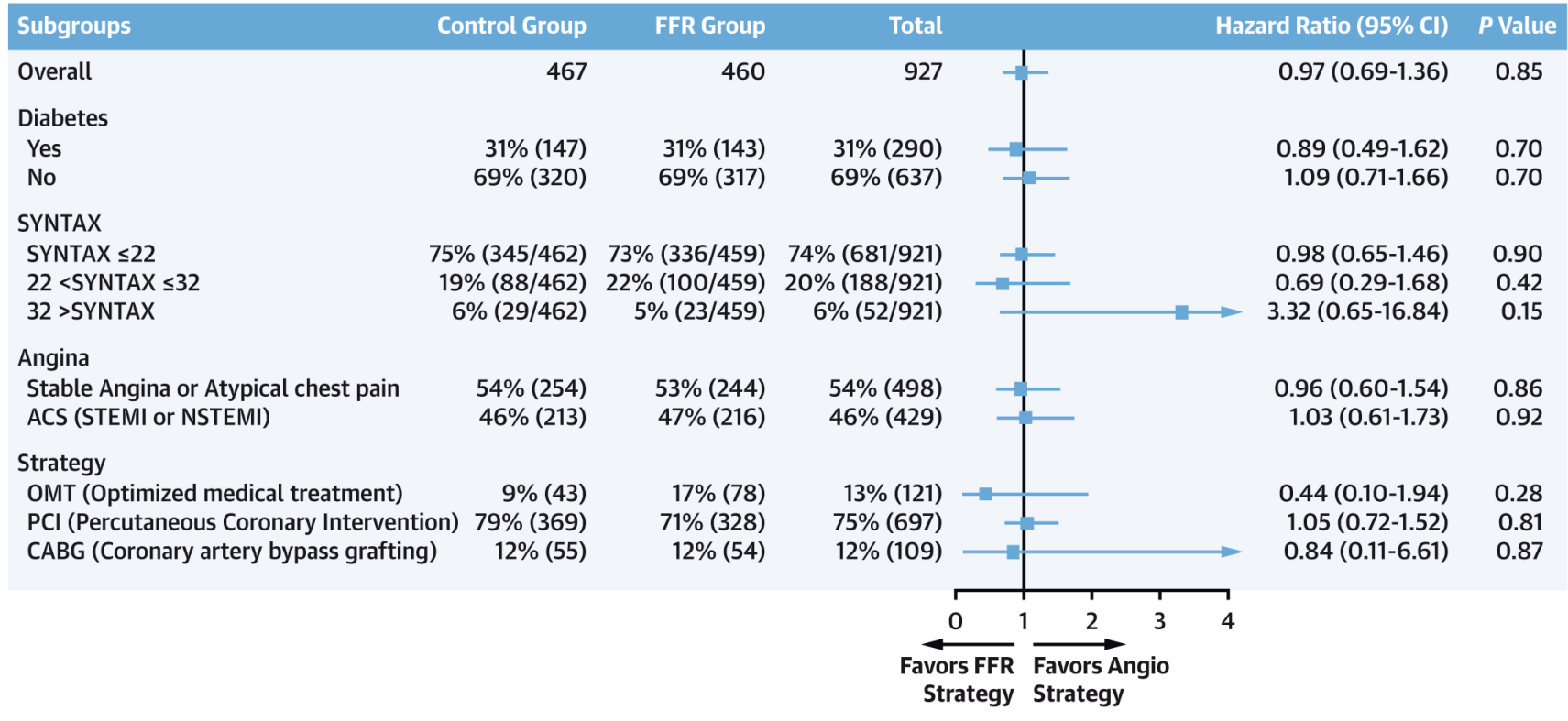

Shown are the hazard ratio estimates for the primary endpoint (death from any cause, myocardial infarction, stroke, and unplanned revascularization) with their $95 \%$ Cls among patients in prespecified subgroups comparing the FFR group with the control group. A hazard ratio $<1.00$ (left panel) is in favor of the FFR group, a hazard ratio $>1.00$ (right panel) is in favor of the control group. Analyses are prespecified in the protocol. ACS = acute coronary syndrome; NSTEMI = non-ST-segment elevation myocardial infarction; OMT = optimal medical treatment; STEMI = ST-segment elevation myocardial infarction; SYNTAX = SYNergy between PCI with TAXUS and Cardiac Surgery; other abbreviations as in Figure 2.

versus traditional angiography assessment (7); however, in case of ACS, clinical impact of FFR is mixed and debatable (16-18). In these studies, both strategies were used by investigators to decide whether or not to revascularize coronary vessels with PCI, without considering surgery. The difference in the findings of these studies compared with the FUTURE trial could be due to a difference in study populations. The majority of previous randomized clinical trials that assessed use of FFR were performed in patients eligible for PCI $(7,13,15-17)$. In all these trials, patients who were eligible for CABG revascularization or had coronary lesions not treatable by PCI were not included. Also, recent phase 2 clinical trials in patients referred to CABG showed that an FFR-based strategy did not significantly improve outcomes at 6-month or 1-year follow-up $(19,20)$.

In contrast, the FUTURE trial was designed to assess whether FFR might help chose the best therapeutic option between PCI, CABG, and medical treatment in an all-comer coronary angiography population. In a smaller group of 350 NSTEMI patients, the FAMOUS-NSTEMI trial compared the impact of FFR on treatment strategy decision making versus angiography alone and showed no difference in MACE rates at 1-year follow-up (8).

The FUTURE trial population had more severe CAD than in previous FFR trials $(7,13,15)$. In our study population, the average SYNTAX score $(19 \pm 8)$ was higher than in the FAME study $(14.5 \pm 9)(7)$. Patients were older and more often diabetic than in the COMPARE-ACUTE (Comparison Between FFR Guided Revascularization Versus Conventional Strategy in Acute STEMI Patients With MVD) or FAMOUSNSTEMI trials $(8,17)$. More than $50 \%$ of our population had 3-vessel disease, which was 2-fold more than in the FAME study or FAMOUS-NSTEMI trial. Moreover, $>1$ in 10 patients in the FUTURE trial had significant left main disease ( $>50 \%$ stenosis), which was a noninclusion criterion in the other studies (7,8,15-17).

It may be possible that, for the most severe CAD patients, global atheroma extension undermines a functional approach with FFR. Thus, in case of very severe disease, as expressed by high SYNTAX scores, the functional impact of significant stenosis assessed as FFR $<0.80$ is less than in case of FFR $<0.80$ in patients with lower SYNTAX score. 


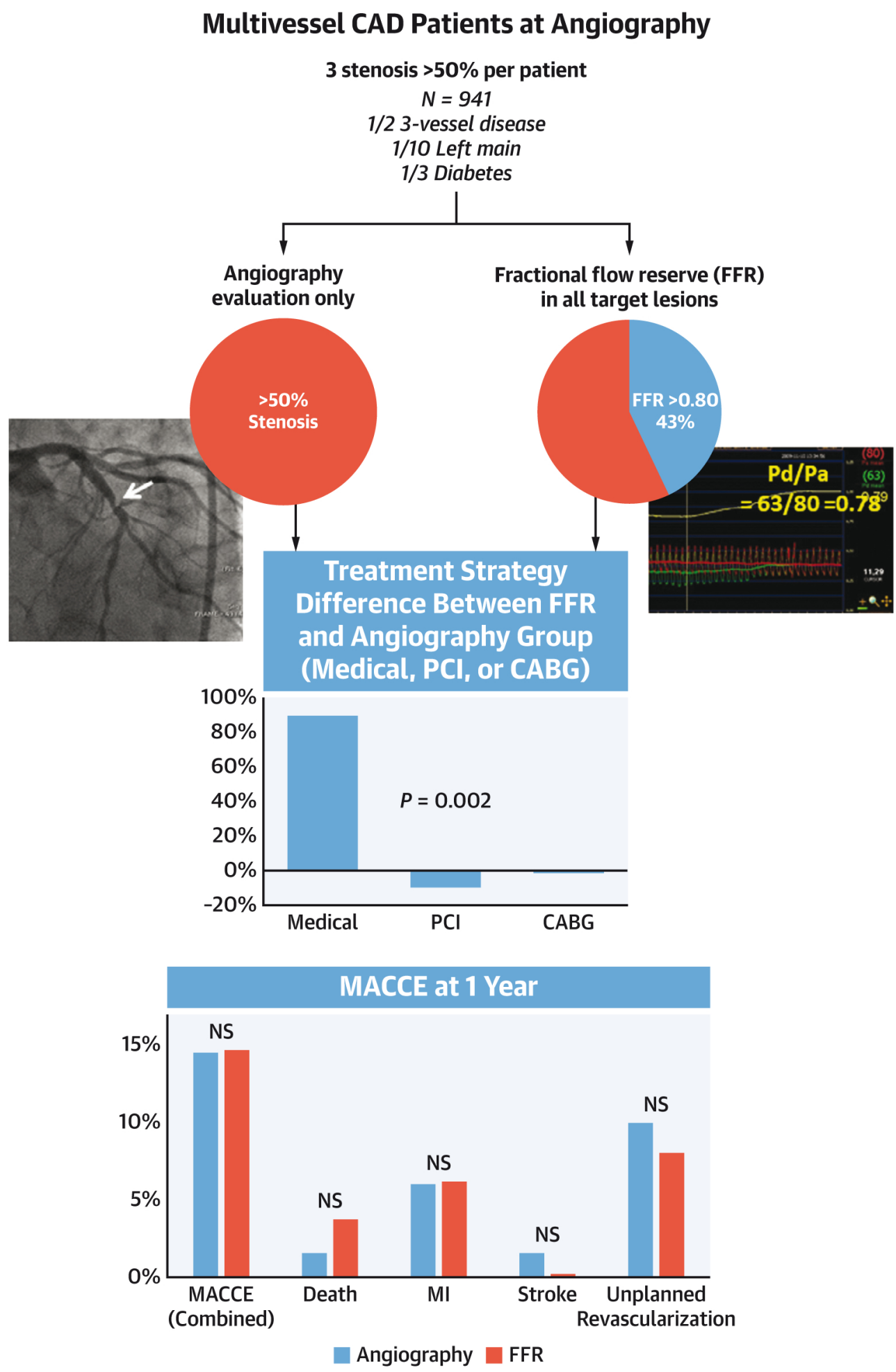

Rioufol, G. et al. J Am Coll Cardiol. 2021;78(19):1875-1885.

The FUTURE (FUnctional Testing Underlying coronary REvascularization) trial compared a fractional flow reserve (FFR)-based strategy with a traditional angiographic strategy in 941 multivessel disease patients. FFR was $>0.80$ in around $40 \%$ of stenosis and nearly doubles the rate of the medical treatment-only strategy. An FFRguided strategy does not modify cardiovascular outcomes at 1 year in comparison with a classical care. CABG = coronary artery bypass grafting; CAD = coronary artery disease; FFR = fractional flow reserve; $\mathrm{MACCE}=$ major adverse cardiac or cerebrovascular events; $\mathrm{MI}=$ myocardial infarction; $\mathrm{NS}=$ not significant; $\mathrm{PCI}=$ percutaneous coronary intervention; $\mathrm{Pd} / \mathrm{Pa}=$ ratio of distal coronary pressure $(\mathrm{Pd})$ on aortic coronary pressure ( $\mathrm{Pa}$ ) under hyperemia giving the $\mathrm{FFR}$ value. 
Routine integration of FFR into the decisionmaking process for patients with obstructive CAD is associated with a significant rate of treatment reclassification, with fewer revascularization procedures $(6-8,13,21,22)$. This impact of FFR has been shown to be safe in registries and randomized controlled trials including PCI-eligible patients. In the present study, FFR use significantly reduced the proportion of revascularized patients, with more patients referred to medical treatment only. This influence of FFR on therapeutic decision making was comparable to the $9.5 \%$ increase in OMT in the FAMOUS-NSTEMI trial (8). Thus, in our multivessel CAD population, FFR significantly increased the proportion of patients treated by medication only 2-fold in comparison with angiography alone. There were $12.6 \%$ fewer stents in the FFR group, but the total number of stents was higher than in other studies using only PCI as the revascularization technique $(7,17)$. This was certainly due to changes in strategy with surgery and certain lesions with FFR $>0.80$ being revascularized at the physician's clinical discretion, the FUTURE trial being a real-life study. Conversely, operators' experience with FFR probably led to less stenting than the expected rate in the angiography group. On overall analysis, the CABG rate did not decrease in the FFR group, but this does not reflect the individual strategy changes between PCI, CABG, and OMT, which ranged between 21\% (8) and $43 \%$ (22), probably accounting for this aspect.

Recently, in multivessel disease patients with low ejection fraction, presence or absence of ischemia did not modify outcome after CABG (23). In the FUTURE trial, exploratory multivariate analysis showed that ejection fraction $<40$ and SYNTAX score $>32$ were independently associated with MACCE rate, but FFR failed to add clinical value in this setting. This suggests that, in the most severe patients with complex coronary lesions, the additive value of myocardial ischemia data provided by FFR may not be always useful.

STUDY LIMITATIONS. Because of the premature recruitment termination after DSMB request, only $54.0 \%$ of the originally planned sample was enrolled, limiting statistical power to demonstrate benefit with an FFR-based treatment strategy. One should, however, note that, despite the lack of statistical power and considering the observed rates of adverse events, there would have been only $3.1 \%$ conditional statistical power to demonstrate any difference between groups if the total number of planned patients $(n=1,728)$ had been recruited. However, the absence of difference might be related to the small differences in overall treatment strategies. In the FFR group, the clinical decision to revascularize some lesions despite a value $>0.80$ might have further reduced the difference between groups. Finally, our study population might not be representative of all multivessel CAD patients.

\section{CONCLUSIONS}

In patients with multivessel coronary artery disease, an FFR-guided strategy nearly doubled the rate of OMT alone and decreased revascularization rates in comparison with angiography alone. The FFR-guided strategy, however, did not significantly influence clinical outcomes at 1 year, which mainly depended on left ventricular function and SYNTAX score. The present study suggests that FFR helps in deciding the most appropriate revascularization strategy but does not per se influence clinical outcome (Central Illustration).

ACKNOWLEDGMENTS The authors of this trial thank all members from the Hospices Civils de Lyon sponsor authorities, namely Muriel Malbezin, Valerie Plattner, Irène Zublena, Marie Lapeyre, and all clinical research associates for their invaluable work on this trial. The authors also thank all members of the different adjudication committees for their expert work, especially Dr Cyrille Bergerot and Dr Brigitte de Breyne. The investigators in the FUTURE (FUnctional Testing Underlying coronary Revascularization) trial are listed in the Supplemental Appendix.

\section{FUNDING SUPPORT AND AUTHOR DISCLOSURES}

This work was supported by an academic grant (Programme Hospitalier de Recherche Clinique National) from the French Government, and the Hospices Civils de Lyon was the academic sponsor, involved in the collection and verification of all the study data. St Jude Medical and Volcano provided $46 \%$ of fractional flow reserve wires without any charge. The authors have reported that they have no relationships relevant to the contents of this paper to disclose.

ADDRESS FOR CORRESPONDENCE: Dr Gilles Rioufol, Department of Hémodynamique et Cardiologie Interventionnelle, Hôpital Cardiologique et Pneumologique Louis Pradel, 28 avenue du Doyen Lépine, 69677 Bron, France. E-mail: gilles.rioufol@univ-lyon1.fr. 
